\title{
Statistical Approach based Optimization for the Application of Chaotic Sequences to Radar
}

\author{
Zouhair Ben Jemaa \\ Laboratoire RISC \\ ENIT, Université de Tunis El Manar \\ Tunis, Tunisia \\ zouhair.benjemaa@enit.rnu.tn
}

\author{
Sylvie Marcos \\ Laboratoire L2S, CNRS UMR8506 \\ CentraleSupelec, Université Paris Sud \\ Paris, France \\ sylvie.marcos@12s.centralesupelec.fr
}

\author{
Safya Belghith \\ Laboratoire RISC \\ ENIT, Université de Tunis El Manar \\ Tunis, Tunisia \\ safya.belghith@enit.utm.tn
}

\begin{abstract}
In this paper, we consider chaotic sequences as an alternative to other sequences in the literature for the design of radar waveforms in multiple-input multiple-output (MIMO) radar. For this aim we here adopt a statistical approach; by considering the codes defining the transmitted waveform as realizations of a random variable we show that a suitable distribution of the random variable can give good codes. As an example we show how the chaotic skew tent map allows to generate deterministic codes having the desired statistical properties and thus makes it possible to obtain a good ambiguity function. These results are confirmed by simulations and compared to those using Gold codes and optimized codes.
\end{abstract}

Index Terms-MIMO radar, chaos-based sequences, ambiguity function, skew tent map, invariant probability density

\section{INTRODUCTION}

In coherent MIMO radar [1] the search for transmitted waveforms leading to the "optimal" ambiguity function (AF), in particular in terms of low side lobes, has been much discussed in the literature [2-7]. Some works are inspired by the field of multi-user communications [2], others aim to synthesize sequences by optimizing certain criteria concerning their auto- and cross-correlation functions [8-12]. Still other studies relate to the joint optimization of the transmit waveform and the receive filter [13] or else consider a multiobjective optimization [14]. This gives rise to a non-linear optimization. However, the sequence-based waveforms generated by the existing methods have drawbacks. Either they are limited in length, or require important calculations, especially when we need a large number of them or we want to add one. In this paper, we propose to consider chaotic sequences as an alternative to other sequences in the literature for the design of radar waveforms. They are random-type sequences generated by nonlinear and recursive deterministic systems controlled with appropriate parameters and initial conditions. These sequences can be easily generated, of any length and in any number. There is no need to store a library or optimize a given function to create a new sequence.

Earlier articles have already suggested the use of chaotic sequences as candidates for the design of radar waveforms [15], [16] and the references inside; in these works the optimization is done by looking for orthogonal sequences having good auto-correlation functions. However, no real theoretical study has shown their interest in a radar system where a Doppler phenomena is added to the auto and crossinterferences. In this paper we propose a statistical approach to exploit the features of chaotic sequences in MIMO radar system. A similar approach has been proposed in [17] for the SIMO context and encouraging results have been found. By considering each code as a realization of a random variable we show that we can build good codes for a radar system if the distribution of the random variable is suitably chosen. For example we show that for sequences generated by the skew tent map and for certain values of its bifurcation parameter, the invariant distribution coincides with the desired distribution.

In section II we introduce the AF and extract the function of interest to be optimized, in section III we study the statistical properties of the latter. In section IV, we show how sequences generated by the skew tent map meet the desired statistical properties established in the previous section. Simulation results illustrate our results in section $\mathrm{V}$ and show a comparison with Gold sequences and the optimized sequences proposed in [3]. The conclusion will summarize the contribution of the paper and present future extension.

\section{THE MIMO RADAR AMBIGUITY FUNCTION}

The MIMO AF corresponding to the reception of signals in the direction $\theta$, for a delay $\tau$ and a Doppler frequency $\nu$ is defined as [1]:

$$
A\left(\theta, \theta_{t}, \tau, \nu\right)=\sum_{m=1}^{M} \sum_{m^{\prime}=1}^{M} \beta_{m, m^{\prime}}\left(\theta, \theta_{t}\right) A_{m, m^{\prime}}(\tau, \nu)
$$

$M$ is the number of waveforms, $\theta_{t}$ is the direction of the target; $\beta_{m, m^{\prime}}\left(\theta, \theta_{t}\right)$ is a function of the antenna elements positions and the wave vectors [7]. In (1),

$$
A_{m, m^{\prime}}(\tau, \nu)=\int s_{m}(t) s_{m^{\prime}}^{*}(t+\tau) e^{j 2 \pi \nu t} d t
$$

is the cross-AF of $s_{m}(t)$ and $s_{m^{\prime}}(t) . s_{m}(t)$ is given by:

$$
s_{m}(t)=\sum_{p=1}^{N_{c}} w_{m, p} u\left(t-(p-1) T_{c}\right)
$$


$N_{c}$ is the length of the sequences $\left\{w_{m, p}\right\}_{p=1 \ldots, N_{c}} ; u(t)$ is a shaping function of duration the pulse repetition interval $T_{c}$. The discretized version of $A_{m, m^{\prime}}(\tau, \nu)$ is

$$
\begin{gathered}
A_{m, m^{\prime}}\left(k T_{c}, \nu\right)=\sum_{p=1}^{N_{c}} \sum_{l=1}^{N_{c}} \\
w_{m, p} w_{m^{\prime}, l}^{*} \int u\left(t-(p-1) T_{c}\right) u\left(t-(l-k-1) T_{c}\right) e^{j 2 \pi \nu t} d t
\end{gathered}
$$

By replacing $l-k$ by $p^{\prime}$ we obtain

$$
\begin{gathered}
A_{m, m^{\prime}}\left(k T_{c}, \nu\right)=\sum_{p=1}^{N_{c}} \sum_{p^{\prime}=1-k}^{N_{c}-k} \\
w_{m, p} w_{, p^{\prime}+k}^{*} \int u\left(t-(p-1) T_{c}\right) u\left(t-\left(p^{\prime}-1\right) T_{c}\right) e^{j 2 \pi \nu t} d t
\end{gathered}
$$

Since $u(t)$ is null outside the interval $\left[0, T_{c}\right]$, the integral vanishes if $p \neq p^{\prime}$ and thus we obtain

$$
\begin{gathered}
A_{m, m^{\prime}}\left(k T_{c}, \nu\right)= \\
\sum_{p=1}^{N_{c}-k} w_{m, p} w_{m^{\prime}, p+k}^{*} \int\left|u\left(t-(p-1) T_{c}\right)\right|^{2} e^{j 2 \pi \nu t} d t
\end{gathered}
$$

By the variable change $t-(p-1) T_{c}$ in $t$ we obtain

$$
\begin{gathered}
A_{m, m^{\prime}}\left(k T_{c}, \nu\right)=\alpha(\nu) R_{m, m^{\prime}}(k, \nu) \\
\alpha(\nu)=\int_{0}^{T_{c}}|u(t)|^{2} e^{j 2 \pi \nu t} d t \\
R_{m, m^{\prime}}(k, \nu)=\sum_{p=1}^{N_{c}-k} \omega_{m, p} \omega_{m^{\prime}, p+k}^{*} e^{j \pi \nu(p-1) T_{c}}
\end{gathered}
$$

In the sequel $R_{m, m^{\prime}}(k, \nu)$ is called the cross-AF for $m^{\prime} \neq m$ and $R_{m}(k, \nu)=R_{m, m}(k, \nu)$ is the auto-AF. The best AF (1) should be maximal for $\theta=\theta_{t}, \tau=0$ and $\nu=0$ and null for $\theta \neq \theta_{t}, \tau \neq 0$ or $\nu \neq 0$. This can be obtained when the auto and cross-correlation functions are such that $R_{m m^{\prime}}(k, \nu)=$ $0, \forall k, \nu$ for $m \neq m^{\prime}$ and $R_{m}(k, \nu)=\delta_{0}(k, \nu), \forall k, \nu$ and $\forall m ; \delta_{0}$ is the two dimentionnal Dirac pulse.

We obtain for phased sequences $\omega_{m, p}=e^{j \pi x_{m, p}}$ :

$$
R_{m m^{\prime}}(k, \nu)=\sum_{p=1}^{N_{c}-k} e^{j \pi\left[x_{m, p}-x_{m^{\prime}, p+k}+\nu(p-1) T_{c}\right]}
$$

If $u(t)$ is the rectangular function of support $\left[0, T_{c}\right], \alpha(\nu)=$ $e^{j \pi \nu T_{c} \frac{\sin \pi \nu T_{c}}{\pi \nu}}$. In practice, the Doppler frequency $\nu$ is usually much smaller than the bandwidth of the probing waveform so that we can safely suppose that $\left|\frac{\sin \pi \nu T_{c}}{\pi \nu}\right| \simeq T_{c}$. It follows that we are going to optimize, for given and fixed values of $m, m^{\prime}$ and $k$, the following expression:

$$
\begin{gathered}
\left|R_{m m^{\prime}}(k, \nu)\right|=\left|\sum_{p=1}^{N_{c}-k} e^{j \pi z_{p}(k)}\right| \\
z_{p}(k)=x_{m, p}-x_{m^{\prime}, p+k}+\nu(p-1) T_{c}
\end{gathered}
$$

It is worth noting that the term $z_{p}(k)$ in (9) that determines the properties of (8) is the summation of a random variable $x_{m, p}-x_{m^{\prime}, p+k}$ and a deterministic term $\nu(p-1) T_{c}$.

In the sequel, we are going to study the statistical properties of $\left|R_{m m^{\prime}}(k, \nu)\right|$ for fixed values of $m, m^{\prime}$ and $k$ for different distributions of $z_{p}(k)$. To do this, we introduce the notations:

$$
\begin{gathered}
S_{n}=\sum_{p=1}^{n} e^{j \pi \Phi_{p}} \\
Z_{n}=\left|S_{n}\right|
\end{gathered}
$$

We obtain (8) when $n=N_{c}-k$ and $\Phi_{p}=z_{p}(k)$.

Different criteria [20] have been considered to analyse objectively the performance of waveforms in MIMO radar application; in most of the cases the correlation properties of the waveforms have been considered independently of the Doppler term. In this work we have taken into account the Doppler term and considered the following criterion

$$
\begin{aligned}
C & =\max _{k \neq 0 ; \nu} G(k, \nu) \\
G(k, \nu) & =\sum_{m, m^{\prime}} R_{m, m^{\prime}}\left(k, \nu T_{c}\right)
\end{aligned}
$$

\section{STATISTICAL PROPERTIES OF $Z_{n}$}

\section{A. Statistics of $Z_{n}$ in the case of i.i.d. argument $\Phi_{P}$}

Let us begin by ignoring the deterministic term in (9) and suppose $\Phi_{p}$ an i. i. d. random variable with a symmetrical distribution about its mean zero. From the central limit theorem for $n$ large enough and by noting $S_{n}=r \exp (j \pi \theta)$, the real and imaginary parts of $S_{n}$ are approximately Gaussian random variables of means $\alpha=E[r \cos (\pi \theta)]=n E\left[\cos \left(\pi \Phi_{p}\right)\right]$ and $\beta=E[r \sin (\pi \theta)]=n E\left[\sin \left(\pi \Phi_{p}\right)\right]$ and variances $s_{1}$ and $s_{2}$, respectively. The distribution of $r=Z_{n}$ is [18]

$$
\rho(r)=2 r \sqrt{s_{1}+s_{2}} e^{-\left(B^{2}+\left(s_{1}+s_{2}\right) r^{2}\right)} I_{0}\left(2 B\left(s_{1}+s_{2}\right) r\right)
$$

$B=\frac{\alpha}{s_{1}+s_{2}} ; I_{0}$ is the modified Bessel function of the $1^{\text {st }}$ kind. Let us consider the following cases.

1) $\Phi_{p}$ uniformly distributed: Let us assume $\Phi_{p}$ uniformly distributed in $[-a, a], 0<a<1$. It follows that $\alpha=n \operatorname{sinc}(a)$ and $\beta=0 . \rho(r)$ is plotted in Figure 1 for $a=1,0.7,0.8$. We can see that for a fixed value of $\mathrm{R}$ the probability that $r<R$ is the largest for the case $a=1$ corresponding to the case of $e^{j \pi \Phi_{p}}$ uniformly distributed on the unit circle. In this case, it is easy to find [18] that $\alpha=0$ and $s_{1}=s_{2}=n / 2$ so that $B=0$ and the distribution of $Z_{n}$ reduces to the Rayleigh distribution $\rho(r)=2 \rho \exp \left(-r^{2}\right)$. This latter will be considered as a reference in the sequel (blue line in Fig1).

2) $\Phi_{p}$ with a triangular distribution on $[-2 b, 2 b], 1 \leq 2 b \leq$ 2: This distribution is here considered since it corresponds to (9) when $x_{m, p}$ and $x_{m^{\prime}, p+k}$ are uniform in $[-b, b]$ and $\nu=0$. Because of the periodicity of $e^{j \pi \Phi_{p}}$ we have $e^{j \pi \Phi_{p}}=e^{j \pi \tilde{\Phi}_{p}}$ where $\tilde{\Phi}_{p}$ is a random variable in $[-1,1]$. The distributions of $\Phi_{p}$ and $\tilde{\Phi}_{p}$ are plotted together in Figure2. We can see that 


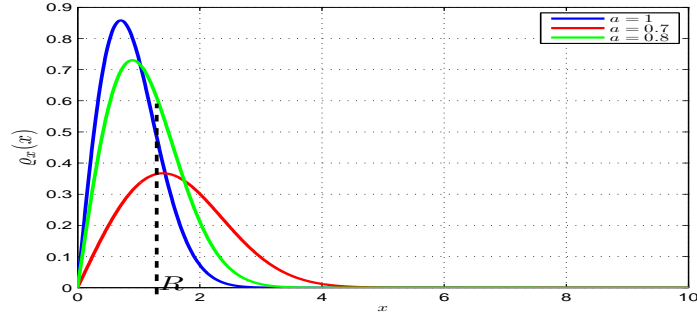

Fig. 1. Distribution of the modulus of $E\left[Z_{n}\right]$

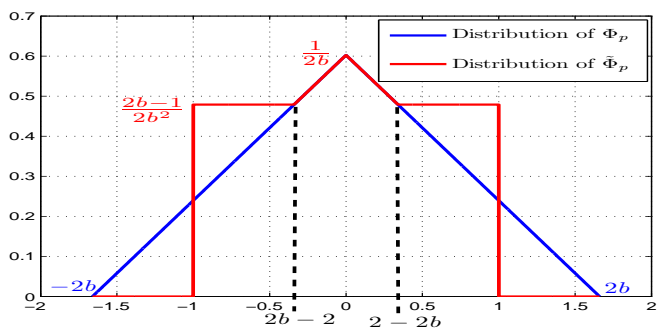

Fig. 2. Distribution of $\Phi_{p}$ and $\tilde{\Phi}_{p}$ in the case of triangular distribution

$\tilde{\Phi}_{p}$ is uniformly distributed in $[-1,1]$ if and only if $b=1$, i.e. we obtain a uniform equivalent distribution in $[-1,1]$ if the distribution of $\Phi_{p}$ is a triangle on $[-2,2]$.

\section{B. Statistics of $Z_{n}$ in the case of $\Phi_{p}=x_{m, p}-x_{m^{\prime}, p+k}$}

Let $\Phi_{p}=x_{m, p}-x_{m^{\prime}, p+k}$. If the $x_{m, p}$ are i.i.d random variables uniformly distributed in $[-a, a], 0<a<1$ the distribution of $\Phi_{p}$ is the triangular function in $[-2 a, 2 a]$. If $2 a<1$ the distribution of $\Phi_{p}$ is triangular within the interval $[-1,1]$ and thus will give bad mean and variance of $Z_{n}$ as explained in the previous section. If $2 a \geq 1$ we retrieve the statistics of $Z_{n}$ described in the previous section, i.e. the Rayleigh distribution is obtained when $a=1$.

C. Statistics of $Z_{n}$ in the case $\Phi_{p}=u_{p}+\nu(p-1) T_{c}$

We here consider the statistics of

$$
\Phi_{p}=u_{p}+\nu(p-1) T_{c}
$$

Here $\Phi_{p}$ is the sum of an i.i.d. random term $u_{p}$ and a deterministic term $\nu(p-1) T_{c}$; since the $u_{p}$ are i.i.d. $E\left[e^{j \pi u_{p}}\right]$ is a constant denoted by $A$ and

$$
E\left[S_{n}\right]=A \sum_{p=1}^{n} e^{j \pi(p-1) \nu T_{c}}
$$

In the case $\nu T_{c}=2 q$ an even integer, $E\left[S_{n}\right]=A \cdot n$. If $A=0$ the real part of $E\left[S_{n}\right]$ is zero; thus $\alpha=B=0$ which are the conditions so that $Z_{n}$ follows a Rayleigh distribution. In the case $\nu T_{c} \neq 2 q$ we can easily show that

$$
\left|\operatorname{Real}\left(E\left[S_{n}\right]\right)\right| \leq|A| n
$$

In this case if $A=0$, the real part of $E\left[S_{n}\right]$ is zero, thus $\alpha=B=0$ and also $\left|Z_{n}\right|$ has a Rayleigh type distribution.

\section{Summary}

We here summarize the previous discussion.

1) The optimization of $\left|R_{m m^{\prime}}(k, \nu)\right|$ in (8) was achieved through the statistical analysis of $Z_{n}(10)-(11)$.

2) The optimization of $Z_{n}$ is obtained if $\Phi_{p}$ in (10) is uniformly distributed in $[-1,1]$ or if it follows the triangular distribution in $[-2,2]$.

3) In the case $\Phi_{p}=z_{p}(k)$ in (9) and $\nu=0$ the optimization is obtained if $x_{m, p}$ is uniformly distributed in $[-1,1]$.

4) As shown in section III.C. the presence of the deterministic term in (9) has no influence if $u_{p}=x_{m, p}-x_{m^{\prime}, p+k}$ follows the triangular distribution in $[-2,2]$ or equivalently if $x_{m, p}$ is uniformly distributed in $[-1,1]$.

In the next section, we illustrate the results summarized above by looking at the example of sequences generated by the skewed tent map and we study the possibility of obtaining such sequences optimizing the $\mathrm{AF}$

\section{Sequences Generated By the SKeW TENT MAP}

Let $x_{p}$ be a sequence generated by $x_{p+1}=T_{\mu}\left(x_{p}\right)$ and an initial condition $x_{0} ; T_{\mu}(x)$ is the piece-wise linear skew tent map defined in $[-1,1]$ by

$$
T_{\mu}(x)=\left\{\begin{array}{ccc}
\frac{2}{\mu-1} x-\frac{1+\mu}{\mu-1} & \text { if } & \mu<x \leq 1 \\
\frac{2}{\mu+1} x-\frac{\mu-1}{\mu+1} & & \text { otherwise }
\end{array}\right.
$$

The invariant distribution of $x_{p}$ is the uniform law in $[-1,1]$ [19] i.e. $x_{p}$ could be considered as a realization of a uniform random variable in $[-1,1]$. The idea is to use such sequences in the MIMO radar system with $x_{m, p+k}=T_{\mu}^{k}\left(x_{m, p}\right)$ for each $m=1, . ., M$, in this case (still $k, m$ and $m^{\prime}$ being fixed):

$$
\Phi_{p}=x_{m, p}-T_{\mu}^{k}\left(x_{m^{\prime}, p+k}\right)
$$

\section{A. Optimisation of the auto-ambiguity function}

For $m=m^{\prime}$ assuming $x_{m, p}$ a random variable we have to consider the statistics of $x_{m, p}-T_{\mu}^{k}\left(x_{m, p}\right)$. Assuming that the $x_{m, p}$ is i.i.d. uniformly distributed in $[-1,1]$ the distribution of $\Phi_{p}=x_{m, p}-T_{\mu}^{k}\left(x_{m, p}\right)$ is triangular in $[-2,2]$ and thus the equivalent distribution $\tilde{\Phi}_{p}$ is uniform in $[-1,1]$. In the Figures 3 and 4 we plotted the distributions of $\Phi_{p}=x_{m, p}-T_{\mu}^{k}\left(x_{m, p}\right)$ in blue line for $\mu=0.1$ and $\mu=0.7$ and for different values of $k$. In red line we plotted the distributions of the equivalent $\tilde{\Phi}_{p}$ of $\Phi$; from the previous discussion $\tilde{\Phi}_{p}$ should be uniform in the interval $[-1,1]$. We can see that after a few iterations the distribution of $\Phi=x_{m, p}-T_{\mu}^{k}\left(x_{m, p}\right)$ tends to the triangular distribution in the interval $[-2,2]$ and thus $\tilde{\Phi}_{p}$ tends to the uniform distribution in the interval $[-1,1]$ for all values of $\mu$, subsequently we have to look for parameter $\mu$ that allows good distributions for the first iterations.

To see the impact of this result we plotted Figure 5 the function (8) for $\mu=0.1$ and $\mu=0.7$ and for $k=1,2,3$. We can see that the case $\mu=0.1$ allows low peaks in (8) yielding a better $\mathrm{AF}$; this is due to the fact that for $\mu=0.1$ the invariant distribution of $x_{m, p}-T_{\mu}^{k}\left(x_{m, p}\right)$ converges more quickly than for the case $\mu=0.7$. This can be observed by comparing the first sub-figures of the Figures 3 and 4 . 

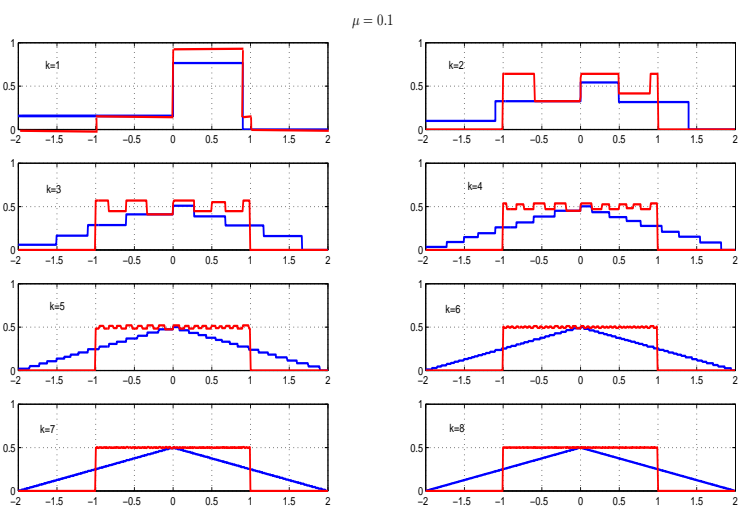

Fig. 3. Probability density of $x-T_{\mu}^{k}(x)$ for $\mu=0.1$
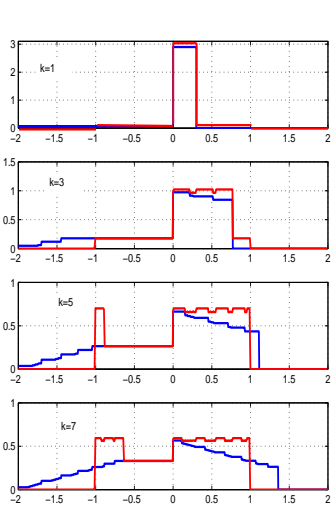

$\mu=0.7$
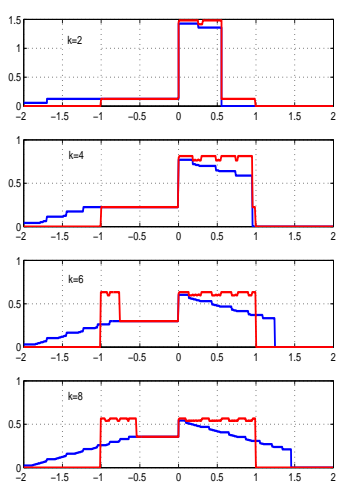

Fig. 4. Probability density of $x-T_{\mu}^{k}(x)$ for $\mu=0.7$

\section{B. Optimisation of the cross-ambiguity function}

Now suppose the initial conditions $x_{m, p}, m=1, \ldots, M$ in (9) independent; thus $x_{m, p+k}$ and $x_{m^{\prime}, p+k}$ are two i.i.d random variables for each couple $m \neq m^{\prime}$. The minimization of (8) is achieved if $\Phi_{p}=x_{m, k}-x_{m^{\prime}, k+p}$ is triangular in $[-2,2]$ which is true for sequences generated by (17) allowing uniform distribution in $[-1,1]$ for all the values of $\mu$.

\section{Simulation Results}

To highlight the previous analysis we plotted in Figures 6 and 7 the AF obtained with the sequences generated by (17) for the cases $\mu=0.1$ and $\mu=0.7, M=3$ and $N_{c}=1023$. We can see that the results agree with the statistical approach developed above i.e. the results are better for $\mu=0.1$.

As a comparison we plotted in Figures 8 and 9 the AF obtained with optimized Multi-CAN and Gold [9] [10] [11] sequences. Subjectively we can see that the Multi-CAN sequences are the best and the Gold sequences are the worst.

We plotted in Figures 10 and 11 the criterion (12) obtained with the chaotic sequences versus the bifurcation parameter $\mu$ for $M=2$ and $M=3$. We can see that these results confirm the theoretical results discussed above: the chaos

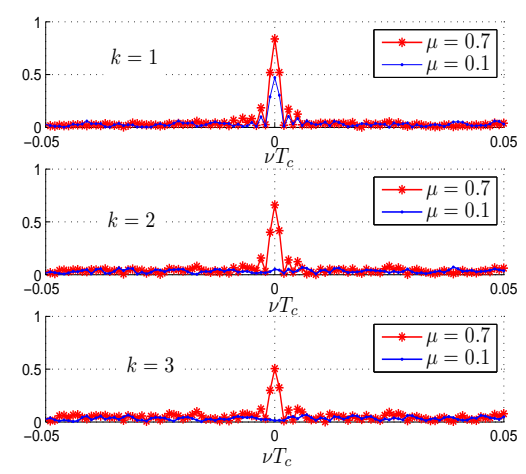

Fig. 5. $|R(k, \nu)|$ for $k=1,2,3$ and $\mu=0.1$ and $\mu=0.7$

based ambiguity function is the best for $\mu=0.1$. In the same figures we plotted the criteria $C$ for Multi-Can sequences; we can see that the MultiCAN sequences are the best. The value of $C$ allowed by Gold sequences is too bad to be plotted in the same figures: for $M=2 C \cong 4$ and for $M=3 C \cong 9$. As explained before the superiority of Multi-CAN sequences with respect to the chaotic ones is due to the fact that the invariant probability density is not uniform in the first iterations.

It is worth noting that the skew tent map is taken as an example to illustrate the approach proposed here; we did not optimize via the choice of chaotic map. This encourages us to further search for other chaotic maps allowing a good invariant distribution in the first iterations as explained in Figs. 3 and 4. This will be done in a future work.

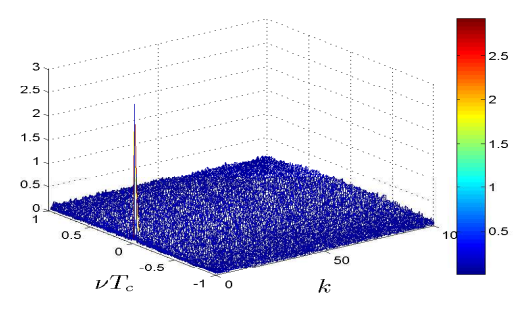

Fig. 6. $G(k, \nu)$ for $\mu=0.1$

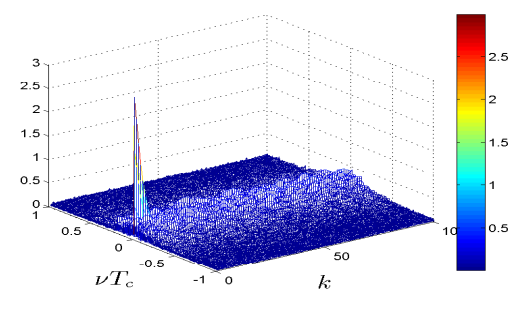

Fig. 7. $G(k, \nu)$ for $\mu=0.7$ 


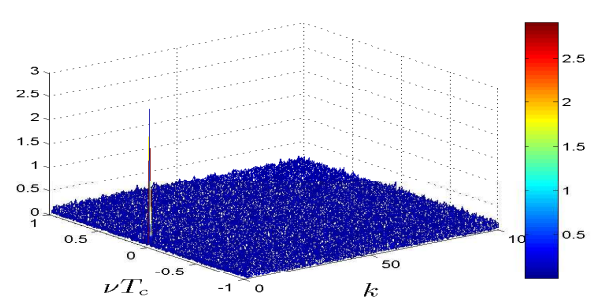

Fig. 8. $G(k, \nu)$ for Multi-CAN sequences

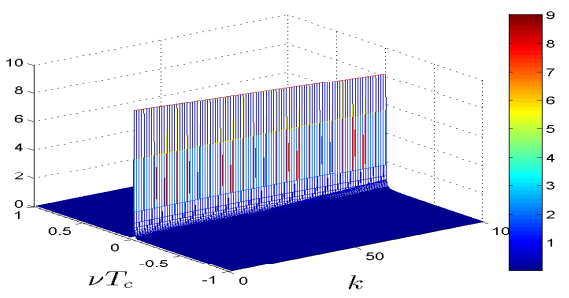

Fig. 9. $G(k, \nu)$ for Gold sequences

\section{CONCLUSION}

In this paper we proposed a statistical approach to seek sequences to use in MIMO radar system; by considering sequences as realizations of a continuous random variable we showed that if the distribution of this random variable is uniform in the interval $[-1,1]$ we could obtain good sequences. As an example we considered chaotic sequences generated by the skew tent map ; indeed these sequences have a stochastic behaviour while being deterministically generated. We showed that statistical features of these sequences are close to the desired ones and that they depend on the bifurcation parameter of the skew tent map. The advantage of chaotic sequences is that they can be generated of any length and in any number. The results obtained are promising and encourage us to take our study further, especially considering other types of chaotic sequences. As demonstrated in this work the drawback of the considered chaotic sequences is due to the first iterations of the corresponding distribution. A method allowing to avoid this weakness has recently been proposed in [21].

\section{REFERENCES}

[1] J. Li, P. Stoica, MIMO radar signal processing. John Wiley and Sons Inc., New Jersey, 2009.

[2] H. Sun, F. Brigui and M. Lesturgie, Analysis and comparison of MIMO radar waveforms, IEEE Radar Conference, pp. 13-17, Cincinnati, OH, USA, May 2014.

[3] Hao He, Waveform Design for Active Sending Systems - A Computational Approach. PhD Thesis, University of Florida, 2011.

[4] F. Arlery, Formes d'onde MSPSR, Traitement et performances associé. PhD Thesis, École doctorale Informatique, Télécommunications et Électronique, Paris, 2017.

[5] U. H. Tan, Méthodologies de conception de formes d'onde pour radars sols- Application au cas du radar MIMO. PhD Thesis, Université ParisSaclay, Paris, 2019.

[6] G. San Antonio, D. R. Fuhrmann, and F. C. Robey, MIMO radar ambiguity functions. IEEE J. Sel. Topics Signal Process, Vol. 1, pp 167-177, June 2007.

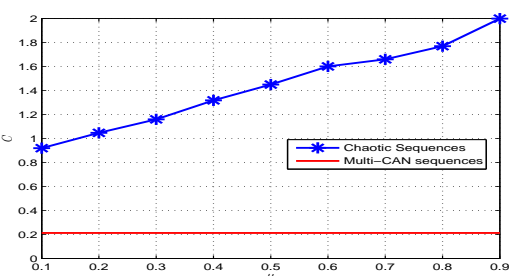

Fig. 10. Criteria $C$ for chaotic and Multi-CAN sequences, $N=1023$, $M=2$

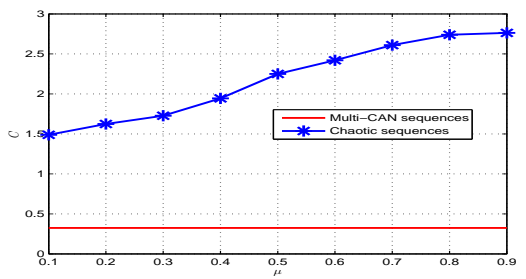

Fig. 11. Criteria $C$ for chaotic and Multi-CAN sequences, $N=1023$, $M=3$

[7] C. Y. Chen, and P. P. Vaidyanathan , MIMO Radar Ambiguity Properties and Optimization Using Frequency-Hopping Waveforms. IEEE Trans. Signal Process, Vol. 56, pp 5926-5936, December 2008.

[8] J. J. Benedetto, I. Konstantinidis, and M. Rangaswamy, "Phase-Coded Waveforms and Their Design," IEEE Signal Processing Magazine, Vol. 26, pp. 22-31, January 2009.

[9] P. Stoica, H. He, and J. Li, On designing sequences with impulse-like periodic correlation, IEEE Signal Processing Letters, Vol. 16, pp. 703706, August 2009.

[10] H. He, P. Stoica and J. Li, Designing unimodular sequence sets with good correlations - including an application to MIMO radar, IEEE Trans. on signal processing, Vol. 57, pp. 4391-4405, November 2009.

[11] F. Arlery, R. Kassab, U. Tan, and F. Lehmann, Efficient gradient method for locally optimizing the periodic/aperiodic ambiguity function, IEEE Radar Conference, Philadelphia, PA, USA, pp. 1-6,May 2016.

[12] U. Tan, C. Adnet, O. Rabaste, F. Arlery, J-P. Ovarlez, and J-P. Guyvarch, Phase code optimization for coherent MIMO radar via a gradient descent, IEEE Radar Conf., Philadelphia, PA, USA, pp. 1-6,May 2016.

[13] D. Ciuonzo, A. De Maio, G. Foglia, M. Piezzo, Intrapulse radarembedded communications via multiobjective optimization, IEEE Trans. on Aerospace and Electronic Syst, Vol. 51, pp. 2960-2974, Oct 2015.

[14] X. Cheng, A. Aubry, D. Ciuonzo, A. De Maio, X. Wang, Robust Waveform and Filter Bank Design of Polarimetric Radar, IEEE Trans. on Aerospace and Electronic Syst, Vol. 53, pp. 370-384, January 2017.

[15] Z. Ben Jemaa, and S. Belghith, Chaotic sequences with good correlation properties for MIMO Radar application, SoftCom Conference, Split, Croatia, pp. 1-6, September 2016.

[16] Y. Jin; H. Wang, W. Jiang, and Z. Zhuang, Complementary-based chaotic phase-coded waveforms design for MIMO radar, IET Radar, Sonar and Navigation, Vol. 7, pp. 371-382, 2013.

[17] Z. Ben Jemaa, Sylvie Marcos and S. Belghith, Optimization of the Radar Ambiguity Function-Application to Chaotic Sequences: Invited Paper, WinCom Conference, Fez, Marocco, 29 October-01 November 2019.

[18] P. Beckmann, Statistical Distribution of the Amplitude and the Phase of a Multiply Scattered Field., Journal of Research of the National Bureau of Standards-Radio Propagation, Vol. 66D, pp 231-240, June 1962.

[19] M. Eisencraft, D.M.Kato, and L.H.A.Monteiro, Spectral properties of chaotic signals generated by the skew tent map., Signal Processing, Vol. 90, pp 385-390, 2010.

[20] G. Galati, and G. Pavan, Waveforms Design for Modern and MIMO Radar, EuroCon 2013, Zagreb, Croatia, pp. 1-6, July 2016.

[21] Z. Ben Jemaa, S. Marcos and S. Belghith, A statistical approach to the optimization of the radar ambiguity function and the chaos-based waveform, Signal Processing, https://doi.org/10.1016/j.sigpro.2020.107649. 